\title{
Unilateral Transforaminal Approach (UTFA) in Percutaneous Vertebroplasty for Osteoporotic Vertebral Compression Fractures: A Novel Puncture Technique
}

wang yefeng ( $\nabla$ wangyefeng0422@sina.com )

Suzhou Municipal Hospital https://orcid.org/0000-0001-6610-103X

\section{Li suoyuan}

Suzhou Municipal Hospital

Cai xiaoqiang

Suzhou Municipal Hospital

Shen jun

Suzhou Municipal Hospital

\section{Zou tianming}

Suzhou Municipal Hospital

\section{Research article}

Keywords: osteoporotic vertebral compression fracture, vertebroplasty, unilateral, bilateral, transforaminal approach, transpedicular approach

Posted Date: January 27th, 2021

DOl: https://doi.org/10.21203/rs.3.rs-154379/v1

License: (c) (1) This work is licensed under a Creative Commons Attribution 4.0 International License.

Read Full License 


\section{Abstract}

Background: Percutaneous vertebroplasty (PVP) via various puncture approaches was an effective minimally invasive treatment for osteoporotic vertebral compression fractures (OVCFs). In recent years, unilateral puncture techniques had been increasingly used with advantages of shorter operation time, lower X-ray exposure. The aim of this study was to explore the safety and efficacy of the unilateral transforaminal approach (UTFA) in PVP for the treatment of OVCFs.

Methods: A retrospective study was designed to review 155 cases of single-level OVCFs from July 2018 to December 2019. We treated 77 patients with PVP via the UTFA and 78 via the bilateral transpedicular approach (BTPA). Operation time, number of intraoperative fluoroscopic X-rays, volume of bone cement injection and distribution of bone cement were recorded. We used Visual Analog Scale (VAS) score and Oswestry disability index (ODI) presurgery and at 1 day, 3 months and last follow-up after surgery to assess clinical outcomes.

Results: The operation was successfully completed in both groups, with no complications of neurovascular injuries or bone cement embolism. UTFA group had significantly shorter operation time and less-frequent fluoroscopy than BTPA group $(P<0.05)$. There were no significant differences between the two groups in volume or distribution of injected bone cement $(P>0.05)$. Postoperative VAS scores and ODI of the two groups were significantly improved over their presurgical values $(P<0.05)$, but there were no significant differences in VAS or ODI at each time point between the two groups $(P>0.05)$.

Conclusions: Both unilateral transforaminal PVP and bilateral transpedicular PVP were safe and effective treatments for OVCFs, but the former approach had the advantages of shorter operation time and less Xray radiation exposure and therefore is worthy of greater clinical application.

\section{Background}

Osteoporosis is a systemic metabolic bone disease characterized by low bone content and microarchitectural deterioration of bone tissue, which increases the risk of fracture [1,2]. With the aging of society, the incidence rate of osteoporotic vertebral-compression fractures (OVCFs) tends to increase year by year [3]. OVCFs are characterized by back pain, spinal deformity and pulmonary dysfunction, which often reduce the patient's quality of life (QoL) and increase the financial burden on patients, the healthcare system and society [4].

Traditional conservative treatments for OVCFs, such as bed rest, analgesic drugs, antiosteoporotic drugs, bracing and physical rehabilitation, can cause respiratory infection, bedsores, acceleration of osteopenia, urinary-tract stones or deep-vein thrombosis $[5,6]$. The alternative of percutaneous vertebroplasty (PVP), which can provide better pain relief, functional recovery and QoL, has become a routine surgical procedure for the treatment of OVCFs $[7,8]$. 
A fractured vertebral body (VB) can be accessed by various puncture methods, including the unilateral and bilateral transpedicular approaches and the unilateral extrapedicular approach $[9,10]$. At this writing, there is still no consensus on the optimal approach, but the unilateral approaches, which have the advantages of shorter operation time, lower radiation dose and less cement leakage, have gradually come to be accepted $[11,12]$. However, the unilateral transpedicular approach has the complications of facet joint damage, pedicle disruption and spinal-canal intrusion [13], and the unilateral extrapedicular approach can injure the lumbar artery [14-16]. To avoid the above-mentioned disadvantages, we used the unilateral transforaminal approach (UTFA) to PVP for the treatment of OVCFs. In this study, we retrospectively analyzed the safety and efficacy of unilateral transforaminal PVP versus bilateral transpedicular PVP for OVCFs.

\section{Methods}

\subsection{Patients}

Patients with painful OVCFs, treated by PVP at our hospital between July 2018 and December 2019, were included in our study. Inclusion criteria for the study were as follows: (1) age $>60$ years; (2) osteoporosis diagnosed by measuring bone mineral density (BMD) using dual-energy X-ray absorptiometry (DEXA), with BMD T-score $\leq-2.5$ [17]; (3) magnetic resonance imaging (MRI) showing low T1- and high T2weighted signals [18]; (4) single-level OVCFs; and (5) <50\% collapse of the VB. Exclusion criteria were as follows: (1) pathological fractures caused by metastatic tumors or hemangiomas; (2) OVCFs with $\geq 2$ levels; (3) spinal-cord compression with neurological impairment; (4) spinal infections; (5) incurable bleeding disorders; (6) severe cardiopulmonary dysfunction; or (7) intolerance of surgery.

A total of 155 patients ( 27 males, 128 females) with a mean age of $77.2 \pm 8.9$ years (range, $60-97$ years) were enrolled in the study. We divided them into two groups according to the puncture approach. Those who underwent PVP via the UTFA were included in the intervention group, and those who underwent PVP via the bilateral transpedicular approach (BTPA) were included in the control group. The details of the two groups are shown in Table 1. There were no significant between-group differences in patient age, sex, BMD, distribution of fractured vertebrae or duration of follow-up (all $P>0.05$ ).

\subsection{Surgical procedure}

The patient was placed in the prone position. Two C-arm X-ray machines were positioned to display anteroposterior (AP) and lateral projections of the fractured vertebra. Senior spine surgeons performed all procedures with patients under local anesthetic with $1 \%$ lidocaine.

UTFA group: The skin entry point was chosen to be $5-7 \mathrm{~cm}$ lateral to the midline at the inferior endplate of the superior vertebra. A trajectory line was drawn between the skin entry point and superolateral area of the fractured vertebra. Under the guidance of the C-arm X-ray machine, a needle gently delivered bone cement via Kambin's triangle to the posterosuperior corner of the vertebra, higher than the sagittal 
superior notch of the pedicle on the lateral image. The needle was placed at the superolateral area of the fractured vertebra, not beyond the medial margin of the ipsilateral pedicle on the AP image. The patient was asked whether radiating pain was felt. Then, the needle's angle and direction were adjusted to penetrate the cortex until the needle tip was positioned at one-third of the anterior part of the VB on the lateral view, and closed to or crossing the midline in AP view. Polymethyl methacrylate (PMMA) cement was prepared at the appropriate viscosity and injected every $0.5-1 \mathrm{ml}$ in both the AP and lateral views. The injection was stopped if the cement reached the posterior quarter of the VB or if cement leakage was noticed. The bone cement needle was not removed until the cement had hardened. Typical case was shown in Fig 1.

BTPA group: Bilateral pedicle projections on the skin were drawn under the AP view. The skin entry point was $0.5-1 \mathrm{~cm}$ lateral to the pedicle projection. Needles were inserted at the 10 o'clock position relative to the left pedicle and at the 2 o'clock position relative to the right pedicle at the craniolateral border of each pedicle. The puncture angle was adjusted under C-arm X-ray fluoroscopy. When the puncture needle tip reached the posterior edge of the $\mathrm{VB}$, care was taken that it did not cross the medial margin of the pedicle in the AP view. Then, the needle was advanced at one-third of the anterior part of the VB in the lateral view. Cement injection procedures were identical to those described for UTFA group.

\subsection{Postoperative treatment}

All patients in both groups were bedridden for $2 \mathrm{~h}$ after surgery. Vital signs, bleeding, sensation and movement of lower extremities were observed. The next day, all patients could rise from bed wearing waist braces; antiosteoporotic drugs were administered, and postoperative spine and chest X-rays were performed. Patients were discharged on the second day after surgery and continued to receive antiosteoporosis treatment.

\subsection{Outcome measures}

Asymmetric distribution of bone cement in a VB can lead to biomechanical imbalance of stress on the VB $[2,19]$. Therefore, we divided the distribution of bone cement in the VB into symmetrical and asymmetrical, per postoperative $\mathrm{X}$-ray results.

We recorded incidences of nerve and blood vessel injury and of bone cement embolism, operation time, number of intraoperative fluoroscopic X-rays, volume of bone cement injected and distribution of bone cement. We used Visual Analog Scale (VAS) score and Oswestry disability index (ODI) presurgery and 1 day, 3 months and last follow-up after surgery to assess clinical outcomes.

\subsection{Statistical analysis}

We used SPSS software version 19.0 (IBM Corp., Armonk, New York, US) for statistical analysis. Measurement data are presented as the mean \pm standard deviation (SD). Intergroup differences were 
evaluated using an independent-sample $t$ test, and intragroup differences using a paired-sample $t$ test. We assessed enumeration data using a $\chi^{2}$ test. A $P$-value of $<0.05$ was considered statistically significant.

\section{Results}

The operation was successfully completed in both groups. There were no spinal-cord or nerve root injuries, no hematomata and no bone cement embolisms.

Differences between the two groups in operation time, number of intraoperative fluoroscopic X-rays, volume of bone cement injected and distribution of bone cement are summarized in Table 2. Operation time and number of intraoperative fluoroscopic X-rays in UTFA group were, respectively, $27.4 \pm 3.0 \mathrm{~min}$ and $16.7 \pm 1.3$ times, which were both lower than those in BTPA group (32.3 $\pm 2.3 \mathrm{~min}$ and $18.2 \pm 1.6$ times, respectively; both $P<0.05)$. There were no statistically significant differences in volume of bone cement injected or distribution of bone cement between the groups (both $P>0.05$ ).

VAS score and ODI were significantly improved after surgery compared with their presurgical values in both groups (both $P>0.05$ ), but there were no statistically significant between-group differences at the same measured time points (both $P>0.05$; see Table 2).

\section{Discussion}

Since PVP was first reported by Galibert in 1987 [20], it has been recommended as an effective minimally invasive treatment for painful OVCFs [21-23]. The standard approach to PVP is the bilateral transpedicular one. However, in recent years, unilateral puncture techniques such as the unipedicular or extrapedicular approach have been increasingly used, as they have the advantages of shorter operation time, lower X-ray exposure and better cost-effectiveness [24-26]. The optimal target location for unilateral PVP is one-third anterior and midline of the VB so that bone cement can diffuse to the unpunctured side, which balances the stress on the VB $[19,27]$. Therefore, the unipedicular approach requires a more aggressive medial-inclination angle, which can lead to complications of facet joint damage, pedicle disruption and spinal-canal intrusion [13, 28]; meanwhile, the extrapedicular approach carries the risk of lumbar-artery injury [14-16]. In this study, we used the unilateral transforaminal approach to PVP for the treatment of OVCFs. We saw none of the above-mentioned complications, and operation time and X-ray radiation exposure were significantly lower than those in bilateral transpedicular PVP.

The intervertebral foramen is located between the deep arch of the inferior vertebral notch of the superior vertebra and the shallow arch of the superior vertebral notch of the inferior vertebra $[29,30]$. The spinal root and vascular plexus course through the anterosuperior midportion of the foramen, immediately inferior to the upper pedicle $[29,31]$. The skin entry point for the unilateral transforaminal approach is at the inferior endplate of the superior vertebra, at the inferior midportion of the foramen. Therefore, the 
unilateral transforaminal approach can anatomically avoid injuring the nerve root and vessel. The operation is performed under local anesthesia so that the patient can be asked about sensations of radiating pain, to avoid the risk of nerve root damage. In the process of puncturing the vertebral surface, the bone needle is placed above the superior notch of the pedicle to avoid injuring segmental vessels [14-16], and not beyond the medial margin of the ipsilateral pedicle, to avoid damaging the spinal cord and dura. In our study, we saw no complications of spinal-cord or nerve root injuries, hematomata or bone cement embolism, which further affirmed the safety of this method.

PVP involves the injection of bone cement into the VB to restore the rigidity and strength of the fractured vertebra, which on average, respectively, require cement fills of $16.2 \%$ and $29.8 \%$ [32]. Röder et al. recommend that at least $4.5 \mathrm{ml}$ bone cement be injected to alleviate pain to a significant degree [33]. Besides cement volume, cement distribution is important to surgical effectiveness. When cement augmentation is limited to the punctured side of the VB only, the rigidity of the unpunctured side remains much weaker, which in turn leads to stress imbalance on the VB and subsequently induces compression and collapse of the weaker side. If cement augmentation crosses the midline, the rigidity of both sides increases to achieve biomechanical balance $[2,19]$. Tan et al. and Zhang et al. both found that when bone cement can fully contact the upper and lower endplates, it can better reduce the risk of vertebral recompression and maintain the height of the VB $[34,35]$. In our study, the volume of bone cement was $5.70 \pm 0.86 \mathrm{ml}$ in UTFA group and $5.82 \pm 0.72 \mathrm{ml}$ in BTPA group, with no statistically significant difference between the groups ( $P>0.05$ ); between-group distribution of bone cement did not differ significantly, either. Although cement was limited to the punctured side only in four cases of UTFA group, we were able to inject it during surgery via the contralateral transpedicular or transforaminal approach to obtain symmetrical distribution. VAS score and ODI in both groups were significantly decreased postsurgery versus presurgery $(P<0.05)$, with no significant differences in VAS score or ODI between the groups $(P>0.05)$. This meant that both unilateral transforaminal and bilateral transpedicular PVP, with similar clinical efficacies, could effectively relieve back pain and improve QoL.

In our study, the UTFA in PVP had the following advantages. First, as a unilateral puncture method, it could simplify the procedure while reducing operation time and X-ray radiation exposure. Second, as the puncture path was made in soft tissue, local anesthesia could reduce intraoperative pain more effectively than in the transpedicular approach. Third, the puncture direction and angle could be adjusted more widely to deliver the bone needle to one-third of the anterior middle region of the VB, which is not restricted by pedicles and is especially suitable for patients with abnormal or small pedicles.

The major limitation of our study was its retrospective design. Therefore, a randomized, controlled prospective study with long-term follow-up is required to confirm these results.

\section{Conclusions}

Both unilateral transforaminal PVP and bilateral transpedicular PVP were safe and effective treatments for OVCFs, but the unilateral transforaminal approach, with the advantages of shorter operation time and 
less X-ray radiation exposure, proved worthy of greater clinical application.

\section{Abbreviations}

PVP: Percutaneous vertebroplasty; OVCFs: Osteoporotic vertebral compression fractures; UTFA: Unilateral transforaminal approach; BTPA: Bilateral transpedicular approach; VAS: Visual Analog Scale; ODI:

Oswestry disability index; QoL: Quality of life; VB: Vertebral body; BMD: Bone mineral density; DEXA: Dualenergy X-ray absorptiometry; MRI: Magnetic resonance imaging; AP: Anteroposterior; PMMA: Polymethyl methacrylate;

\section{Declarations}

\section{Ethics approval and consent to participate}

This study was approved by the Ethics Committees of the Affiliated Suzhou Hospital of Nanjing Medical University (Suzhou, China), and all patients signed written informed consent.

\section{Consent for publication}

Not applicable.

\section{Availability of data and materials}

The datasets analysed during the current study are available from the corresponding author on reasonable request.

\section{Competing interests}

The authors declare that they have no competing interests.

\section{Funding}

Not applicable.

\section{Authors' contributions}

Cai XQ, Shen J and Zou TM contributed to the design of the study. Wang YF and Li SY collected the data. Wang YF, Shen J and Zou TM performed the data management. Wang YF, Li SY and Shen J performed the data analyses and were in charge of data interpretation. Wang YF wrote the manuscript. All authors have read and approved the final manuscript.

\section{Acknowledgements}

Not applicable. 


\section{References}

1. Choi SH. Kim DY, Koo JW. Lee SG, Jeong SY. Kang CN. Incidence and Management Trends of Osteoporotic Vertebral Compression Fractures in South Korea: A Nationwide Population-Based Study. Asian Spine J. 2020;14(2):220-8. doi: 10.31616/asj.2019.0051.

2. Zhong R. Liu J, Wang R. Liu Y, Chen B. Jiang W, Mao K. Tang P. Unilateral curved versus bipedicular vertebroplasty in the treatment of osteoporotic vertebral compression fractures. BMC Surg. 2019;19(1):193. doi: 10.1186/s12893-019-0653-y.

3. Yang D. Zhang Y, Ma X. Huo L, Li L. Gao Y. Resources utilisation and economic burden of percutaneous vertebroplasty or percutaneous kyphoplasty for treatment of osteoporotic vertebral compression fractures in China: a retrospective claim database study. BMC Musculoskelet Disord. 2020;21(1):255. doi: 10.1186/s12891-020-03279-1.

4. Svedbom A. Alvares L, Cooper C. Marsh D, Ström O. Balloon Kyphoplasty Compared to Vertebroplasty and Non-Surgical Management in Patients Hospitalised with Acute Osteoporotic Vertebral Compression Fracture-A UK Cost-Effectiveness Analysis. Osteoporos Int. 2013;24(1):355-67. doi: 10.1007/s00198-012-2102-y.

5. Yang H. Liu T, Zhou J. Meng B, Wang G. Zhu X. Kyphoplasty versus vertebroplasty for painful osteoporotic vertebral compression fractures-which one is better? A systematic review and metaanalysis. Int J Spine Surg. 2013;7:e45-57. doi: 10.1016/j.ijsp.2013.03.001.

6. Zhao WT. Qin DP, Zhang XG. Wang ZP, Tong Z. Biomechanical effects of different vertebral heights after augmentation of osteoporotic vertebral compression fracture: a three-dimensional finite element analysis. J Orthop Surg Res. 2018;13(1):32. doi: 10.1186/s13018-018-0733-1.

7. Pan M. Ge J, Li Q. Li S, Mao H. Meng B, Yang H. Percutaneous vertebral augmentation in special Genant IV osteoporotic vertebral compression fractures. J Orthop Translat. 2019;20:94-9. doi: 10.1016/j.jot.2019.07.002.

8. Ong KL. Beall DP, Frohbergh M. Lau E, Hirsch JA. Were VCF patients at higher risk of mortality following the 2009 publication of the vertebroplasty "sham" trials? Osteoporos Int. 2018;29(2):37583. doi: 10.1007/s00198-017-4281-z.

9. Tam AL. Mohamed A, Pfister M. Chinndurai P, Rohm E. Hall AF, Wallace MJ. C-arm cone beam computed tomography needle path overlay for fluoroscopic guided vertebroplasty. Spine (Phila Pa 1976). 2010;35(10):1095-9. doi: 10.1097/BRS.0b013e3181bc39c6.

10. Chen C. Bian J, Zhang W. Zhang W, Zhao C. Wei H. Unilateral versus bilateral vertebroplasty for severe osteoporotic vertebral compression fractures. J Spinal Disord Tech. 2014 Dec,27(8):E301-4. doi: 10.1097/BSD.0000000000000118.

11. Yan L. He B, Guo H. Liu T, Hao D. The prospective self-controlled study of unilateral transverse process-pedicle and bilateral puncture techniques in percutaneous kyphoplasty. Osteoporos Int. 2016;27(5):1849-55. doi: 10.1007/s00198-015-3430-5. 
12. Yan L. Jiang R, He B. Liu T, Hao D. A comparison between unilateral transverse process-pedicle and bilateral puncture techniques in percutaneous kyphoplasty. Spine (Phila Pa 1976). 2014;39(26 Spec No.):B19-26. doi: 10.1097/BRS.0000000000000493.

13. Erkan S. Wu C, Mehbod AA. Cho W, Transfeldt EE. Biomechanical comparison of transpedicular versus extrapedicular vertebroplasty using polymethylmethacrylate. J Spinal Disord Tech. 2010;23:180-5. doi: 10.1097/BSD.0b013e31819c48a4.

14. Heo DH. Cho YJ. Segmental artery injury following percutaneous vertebroplasty using extrapedicular approach. J Korean Neurosurg Soc. 2011;49(2):131-3. doi: 10.3340/jkns.2011.49.2.131.

15. Puri AS. Colen RR, Reddy AS. Groff MW, DiNobile D. Killoran T, Nikolic B. Thomas AJ. Lumbar artery pseudoaneurysm after percutaneous vertebroplasty: a unique vascular complicationJ Neurosurg Spine. 2011;14(2):296-9. doi: 10.3171/2010.10.SPINE1082.

16. Liu L. Cheng S, Wang Q. Liang Q, Liang Y. Jin W, Zhou Q. Wang Z. PLoS One. 2019;14(3):e0213164. doi: 10.1371/journal.pone.0213164.

17. Keaveny TM. Clarke BL, Cosman F. Orwoll ES, Siris ES. Khosla S, Bouxsein ML. Biomechanical Computed Tomography analysis (BCT) for clinical assessment of osteoporosis. Osteoporos Int. 2020;31(6):1025-48. doi: 10.1007/s00198-020-05384-2.

18. Xu W. Wang S, Chen C. Li Y, Ji Y. Zhu X, Li Z. Correlation analysis between the magnetic resonance imaging characteristics of osteoporotic vertebral compression fractures and the efficacy of percutaneous vertebroplasty: a prospective cohort study. BMC Musculoskelet Disord. 2018;19:114. doi: 10.1186/s12891-018-2040-8.

19. Chen BL. Li YQ, Xie DH. Yang XX, Zheng ZM. Comparison of unipedicular and bipedicular kyphoplasty on the stiffness and biomechanical balance of compression fractured vertebrae. Eur Spine J. 2011 Aug;20(8):1272-80. doi: 10.1007/s00586-011-1744-3.

20. Galibert P. Deramond H, Rosat P. Gars DL. Preliminary note on the treatment of vertebral angioma by percutaneous acrylic vertebroplasty. Neurochirurgie. 1987;33(2):166-8.

21. Hinde K. Maingard J, Hirsch JA. Phan K, Asadi H. Chandra RV. Mortality Outcomes of Vertebral Augmentation (Vertebroplasty and/or Balloon Kyphoplasty) for Osteoporotic Vertebral Compression Fractures: A Systematic Review and Meta-Analysis. Radiology. 2020;295(1):96-103. doi: 10.1148/radiol.2020191294.

22. Yang W. Song J, Liang M. Cui H, Chen H. Yang J. Functional Outcomes and New Vertebral Fractures in Percutaneous Vertebroplasty and Conservative Treatment of Acute Symptomatic Osteoporotic Vertebral Compression Fractures. World Neurosurg. 2019;131:e346-52. doi:10.1016/j.wneu.2019.07.153.

23. Lou S. Shi X, Zhang X. Lyu H, Li Z. Wang Y. Percutaneous vertebroplasty versus non-operative treatment for osteoporotic vertebral compression fractures: a meta-analysis of randomized controlled trials. Osteoporos Int. 2019;30(12):2369-80. doi: 10.1007/s00198-019-05101-8.

24. Cui Z. Tian Y, Feng H. Yang Z, Liu Z. Unilateral Versus Bilateral Balloon Kyphoplasty for Osteoporotic Vertebral Compression Fractures: A Systematic Review of Overlapping Meta-analyses. Pain 
Physician. 2019;22(1):15-28.

25. Xu JC. Wu GH, Zhou LL. Yang XJ, Liu JT. Two unilateral puncturation comparative analyses of multiple-level fresh osteoporotic vertebral body compression fractures treated with percutaneous vertebroplasty guided by C-arm fluoroscopy or in senile patients. Eur Rev Med Pharmacol Sci. 2017;21(7):1456-61.

26. Yin P. Ji Q, Wang Y, et al Percutaneous kyphoplasty for osteoporotic vertebral compression fractures via unilateral versus bilateral approach: A meta-analysis. J Clin Neurosci. 2019;59:146-54. doi:10.1016/j.jocn.2018.10.112.

27. Wang H. Hu P, Xu W, et al Unilateral percutaneous kyphoplasty for lumbar spine: A comparative study between transverse process-pedicle approach and conventional transpedicular approach. Medicine. 2020;99(17):e19816. doi:10.1097/MD.0000000000019816.

28. Wang LM. Liu FY, Lu K. Liu Z, Hou SB. Sun XZ. Modified extrapedicular kyphoplasty for the treatment of lumbar compression fracture: Case report and technical note. Medicine. 2020;99(6):e19053. doi:10.1097/MD.0000000000019053.

29. Gkasdaris G. Tripsianis G, Kotopoulos K. Kapetanakis S. Clinical anatomy and significance of the thoracic intervertebral foramen: A cadaveric study and review of the literature. J Craniovertebr Junction Spine. 2016;7(4):228-35. doi:10.4103/0974-8237.193266.

30. Gkasdaris G. Hourmouzi D, Chaniotakis C, et al CT Assessment of the in vivo Osseous Lumbar Intervertebral Foramen: a Radiologic Study with Clinical Applications. Maedica (Buchar). 2018;13(4):294-304. doi:10.26574/maedica.2018.13.4.294.

31. Nascene DR. Ozutemiz C, Estby H. McKinney AM, Rykken JB. Transforaminal Lumbar Puncture: An Alternative Technique in Patients with Challenging Access. AJNR Am J Neuroradiol. 2018;39(5):98691. doi:10.3174/ajnr.A5596.

32. Molloy S. Mathis JM, Belkoff SM. The effect of vertebral body percentage fill on mechanical behavior during percutaneous vertebroplasty. Spine (Phila Pa 1976). 2003;28(14):1549-54.

33. Röder C. Boszczyk B, Perler G. Aghayev E, Külling F. Maestretti G. Cement volume is the most important modifiable predictor for pain relief in BKP: results from SWISSspine, a nationwide registry. Eur Spine J. 2013;22(10):2241-8. doi:10.1007/s00586-013-2869-3.

34. Zhang Z. Jiao $\mathrm{F}$, Huang $\mathrm{H}$, et al. A second puncture and injection technique for treating osteoporotic vertebral compression fractures. J Orthop Surg Res. 2019;14(1):413. Published 2019 Dec 5. doi:10.1186/s13018-019-1498-x.

35. Tan L. Wen B, Guo Z. Chen Z. The effect of bone cement distribution on the outcome of percutaneous Vertebroplasty: a case cohort study. BMC Musculoskelet Disord. 2020;21(1):541. Published 2020 Aug 13. doi:10.1186/s12891-020-03568-9.

\section{Tables}

Due to technical limitations, table $1 \& 2$ is only available as a download in the Supplemental Files section. 


\section{Figures}

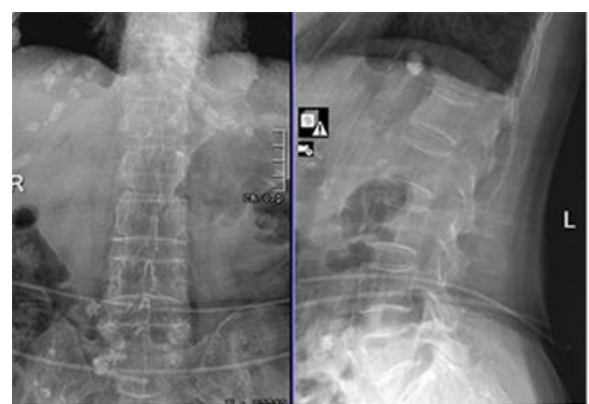

a

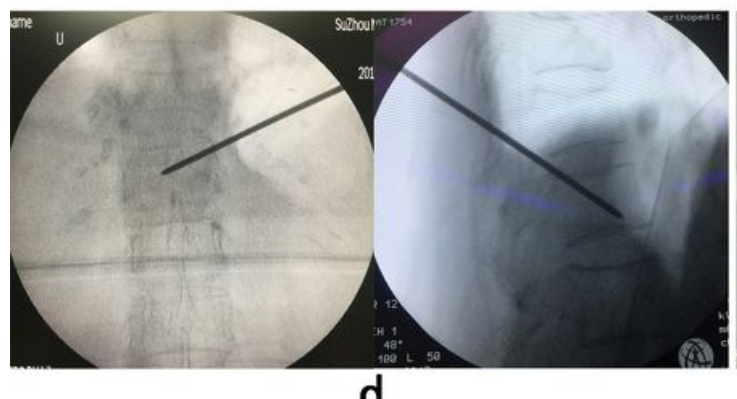

d

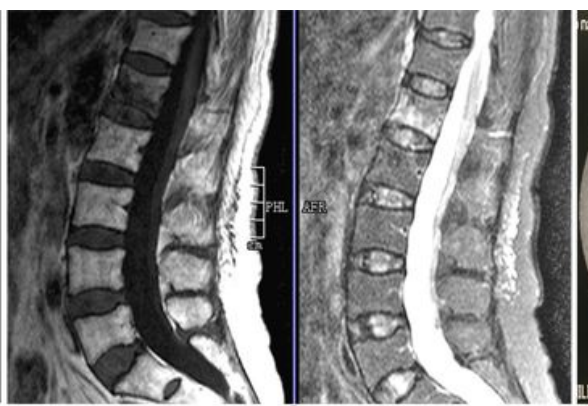

b

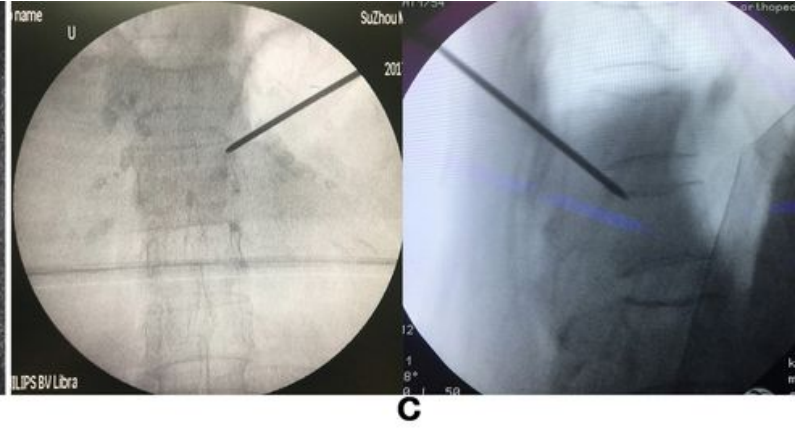

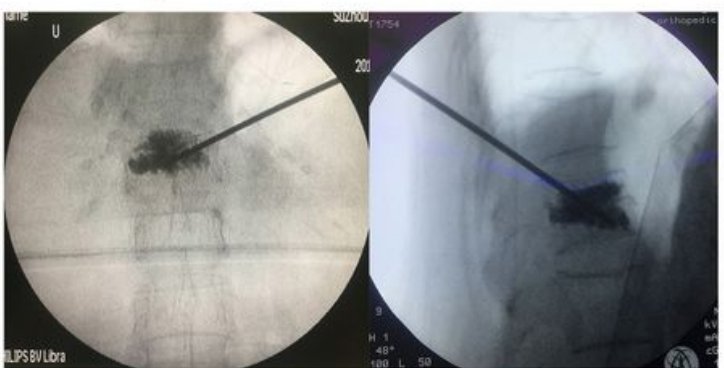

e

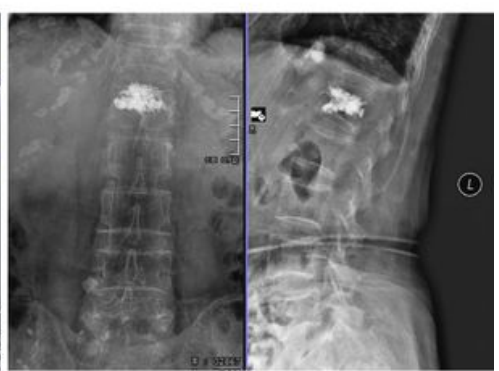

f

\section{Figure 1}

A 81 years old female patient was treated with PVP via unilateral transforaminal approach. a, b:

Preopreaive x-ray (a) and MRI (b) showed acute L1 OVCF. c, d, e: The process of unilateral transforaminal PVP for L1 OVCF: c The needle tip was located at the superolateral area of the VB on the AP image and at the posterosuperior side of the vertebra on the lateral image. $d$ The needle tip crossed the midline on the AP image to one-third of the VB on lateral view. e Cement augmentation crossed the midline and filled the unpunctured side. f: X-ray on 1 day after operation showed symmetrical cement distribution.

\section{Supplementary Files}

This is a list of supplementary files associated with this preprint. Click to download.

- Table.pdf 